\title{
Uroplakin II outperforms uroplakin III in diagnostically challenging settings
}

\author{
Steven C Smith, ${ }^{*}$ Sambit K Mohanty, ${ }^{*}$ Lakshmi P Kunju, ${ }^{1}$ Elena Chang, Fai Chung, \\ Jason C Carvalho, Gladel P Paner, ${ }^{2}$ Donna E Hansel, ${ }^{3}$ Daniel J Luthringer, Mariza N de \\ Peralta-Ventrurina \& Mahul B Amin \\ Department of Pathology and Laboratory Medicine, Cedars-Sinai Medical Center, Los Angeles, CA, USA, ${ }^{1}$ Department of \\ Pathology, University of Michigan School of Medicine, Ann Arbor, MI, USA, ${ }^{2}$ Department of Pathology, University of \\ Chicago, Chicago, IL, USA, and ${ }^{3}$ Department of Pathology, University of California, San Diego, La Jolla, CA, USA
}

Date of submission 4 November 2013

Accepted for publication 27 December 2013

Published online Article Accepted 2 January 2014

Smith S C, Mohanty S K, Kunju L P, Chang E, Chung F, Carvalho J C, Paner G P, Hansel D E, Luthringer D J, de Peralta-Ventrurina M N, Amin M B

(2014) Histopathology 65, 132-138

\section{Uroplakin II outperforms uroplakin III in diagnostically challenging settings}

Aims: We performed a head-to-head comparison of an antibody against uroplakin III (UP3) and a new uroplakin II (UP2) antibody that remains untested in diagnostically challenging settings.

Methods and results: We immunostained high-grade bladder neck carcinomas $(n=35)$, high-grade upper tract urothelial carcinomas (UC) and renal carcinomas $(n=85)$, metastases of UC $(n=30)$ and a multicancer tissue microarray $(n=88)$ for UP3 and UP2, and scored staining intensity and proportion. UP3 showed membranous plaque-like expression, while UP2 staining showed both membranous and cytoplasmic positivity. Significantly greater intensity $(P=0.003)$ and proportion $(P=0.03)$ of staining was noted for UP2 among bladder neck lesions, with UP2 staining show- ing greater sensitivity (63\% versus 19\%) and similar specificity (95\% versus $100 \%$ ) for UC over prostate carcinoma $(P=0.02)$. Among upper tract lesions, UP2 staining showed greater intensity and proportion than UP3 (both $P<0.001$ ), including improved sensitivity (68\% versus $23 \%$ ) and equal specificity (both 100\%) for UC $(P=0.006)$. Among UC metastases, UP2 staining showed greater intensity and proportion (both $P<0.001)$ with higher sensitivity (73\% versus $37 \%$, respectively, $P=0.001)$. Of 88 additional cases tested, no non-urothelial cases stained for either UP.

Conclusions: The UP2 antibody outperforms the UP3 antibody, including in diagnostically challenging settings, and is a useful addition to the armamentarium of biomarkers for $\mathrm{UC}$.

Keywords: biomarkers, immunohistochemistry, uroplakin, urothelial carcinoma

\section{Introduction}

The urothelial lining of the pelvicalyceal mucosa of the kidney, the ureter, the urinary bladder and the proxi-

Address for correspondence: M B Amin, MD, Professor and Chairman, Department of Pathology and Laboratory Medicine, Cedars-Sinai Medical Center, 8700 Beverly Boulevard, South Tower, Room 8709, Los Angeles, CA 90048, USA. e-mail: aminm@cshs.org

*These authors contributed equally to this study. mal urethra comprises a stratified epithelium of five to seven cell layers; these strata proceed from a basal cell population, through intermediate cells, to an umbrella cell population lying immediately at the luminal surface. The latter umbrella cells produce specialized urothelial plaques composed of uroplakins Ia, Ib, II and IIIa, which occupy the luminal surface of the epithelium, and cytoplasmic vesicles from where they may be cycled to the surface during the expansion and contraction cycle of the urothelium, as reviewed recently. ${ }^{1}$ 

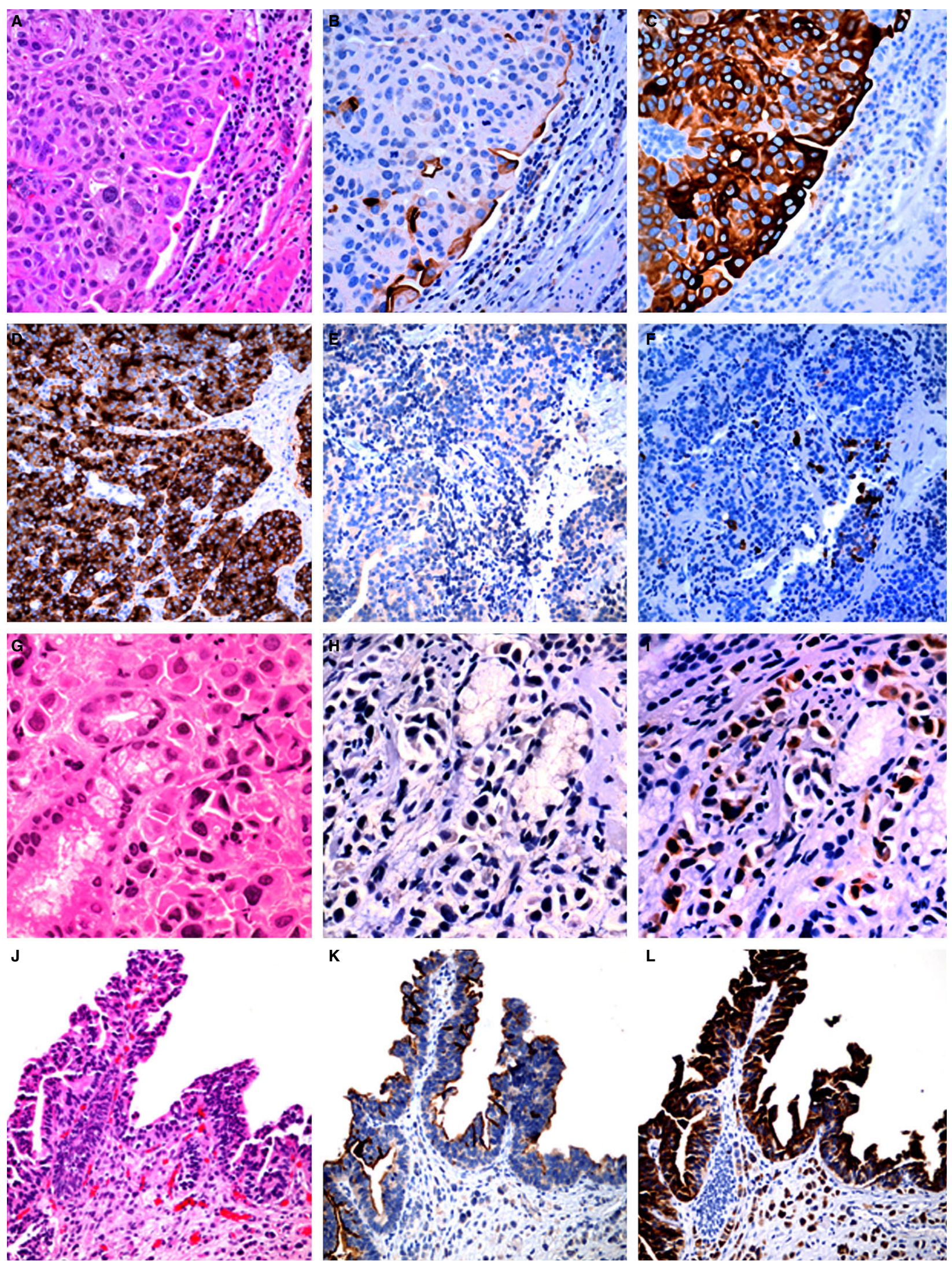
Conventional markers of urothelial carcinomas (UC) include p63, high molecular weight cytokeratin and cytokeratin $5 / 6$, also expressed in squamous lesions, and coexpression of cytokeratins 7 and 20, which is neither highly sensitive nor specific. Improved markers of urothelial histogenesis include GATA3 and S100P. ${ }^{2}$ However, recent studies have demonstrated significant rates of GATA3 positivity in primary and metastatic ductal and lobular carcinomas of the breast, cutaneous basal cell carcinomas, trophoblastic neoplasms, yolk sac tumours, mesotheliomas, salivary gland carcinomas and pancreatic ductal carcinomas, ${ }^{3}$ prompting greater circumspection regarding its specificity. Also, significant positivity for S100P has been shown in pancreaticobiliary lesions. ${ }^{4}$ Several years ago, staining for uroplakin III (UP3) was introduced as a highly specific and moderately sensitive marker of urothelial carcinoma, showing 'plaque-like' membranous expression. ${ }^{5}$ Sensitivities reported were in the range of $50-60 \%, 5,6$ although we and others have observed lower sensitivities, ${ }^{7,8}$ especially in invasive or metastatic lesions. ${ }^{8,9}$ None the less, this marker shows exquisite specificity, with no staining observed in hundreds of non-urothelial lesions. ${ }^{5,8}$

Recently, a new monoclonal antibody has been raised against another uroplakin, uroplakin II (UP2). UP2 is a smaller, related protein ( 15 versus $47 \mathrm{kDa})$, which dimerizes with uroplakin Ia rather than $\mathrm{Ib}$, contains a more limited cytoplasmic domain, and is not glycosylated. ${ }^{1}$ Preliminary comparisons using primary urothelial carcinomas and other cancers suggest that UP2 has greater sensitivity than UP3, with preserved specificity; ${ }^{10}$ these observations have not been tested in relevant diagnostic settings where identification of UC, as opposed to other lesions in the differential, may result in fundamental therapeutic differences. Thus, we tested UP2 and UP3 antibodies 'head-to-head' in diagnostically challenging settings: (i) high-grade carcinomas involving the bladder neck, (ii) high-grade carcinomas of the upper urothelial tract and (iii) metastatic UC.

\section{Materials and methods}

COHORTS TESTED

A retrospective cohort of archival tissues was assembled for testing in three diagnostically challenging settings. The first was a cohort of whole sections of high-grade UC and high-grade prostatic adenocarcinomas (PCa) involving the bladder neck $(n=35)$. The second cohort included a tissue microarray cohort, previously assembled and reported ${ }^{11}$ to comprise aggressive ( $>$ pT3) high-grade carcinomas of the upper tract $(n=70)$, plus whole tissue sections of upper tract UC $(n=10)$ and collecting duct carcinomas (CDC) $(n=5)$. The third cohort included whole sections of metastatic deposits of UC $(n=30)$. Finally, additional descriptive studies were performed on whole sections of rare variants of UC $(n=30)$ and a tissue microarray (TMA) of representative examples of neoplasms of varying histologies $(n=88)$, including adenocarcinomas $(n=25)$, neuroendocrine tumours $(n=15)$, squamous cell carcinomas $(n=10)$, renal and germ cell tumours (each $n=6$ ), salivary gland and thyroid tumours (each $n=5)$, other carcinomas $(n=4)$, paragangliomas $(n=3)$, urothelial carcinomas $(n=2)$ and other $(n=7)$. These cases were selected across a wide anatomical distribution, including respiratory $(n=15)$, gastrointestinal $(n=25)$, genitourinary and gynaecological $(n=36)$ and endocrine $(n=12)$ organs.

\section{M M U N OH I S T O C HEM I S T R Y}

For both UP2 (clone BC21 prediluted; Biocare Medical, Concord, CA, USA) and UP3 (clone AU-1 prediluted; Cell Marque, Rocklin, CA, USA), immunohistochemistry (IHC) was performed on a Benchmark Ultra (Ventana, Tucson, AZ, USA) autostainer in the Cedars-Sinai Medical Center Clinical Immunohistochemistry core facility. IHC used integrated heat-induced epitope retrieval in high $\mathrm{pH} \mathrm{CC} 1$ buffer, with visualization using the Ultraview DAB detection system (Ventana) and Mayer's haematoxylin counterstain.

Figure 1. Representative examples of UP2 and UP3 immunostaining. A-C, A high-grade upper tract urothelial carcinoma (UC) (A), shows $2+$ intensity and multifocal (2+) staining for UP3 (B), and intense (3+), diffuse (3+) UP2 expression (C). D-F, A Gleason score 4+5 adenocarcinoma of the bladder neck with diffuse prostate-specific membrane antigen positivity, (D), shows negative staining for UP3 (E), and intense (3+), focal (1+) UP2 expression (F). This was the only case of aberrant expression of UP2 in >160 non-urothelial carcinomas tested. G-H, A duodenal metastasis in a patient with a long history of UC shows poorly differentiated UC with extensive lymphatic involvement (G) shows negative staining for UP3 (H), and moderate (2+), multifocal (2+) UP2 expression (I). K, A bladder UC variant with 'villoglandular' morphology (exophytic component) and subjacent invasive plasmacytoid UC morphology (J) shows moderate (2+), multifocal (2+) UP3 expression in the villoglandular areas and negative UP3 expression in plasmacytoid areas (K). UP2 staining is intense (3+) and diffuse (3+) in both components $(\mathbf{L})$. 


\section{E V A L U A TI O N OF IM MUNOHISTOCHEMISTR Y}

Immunostain intensity and proportion were scored independently by two pathologists (S.C.S. and S.K.M.), with discordances resolved in consensus with a senior genitourinary pathologist (M.B.A.). Intensity was scored as 0 (negative), 1 (weak), 2 (moderate) and 3 (intense), while proportion was scored as 0 (negative), 1 (focal), 2 (multifocal) and 3 (diffuse). Focal staining was defined arbitrarily as the minimum proportion that would reasonably considered as positive if it were encountered prospectively in a diagnostically challenging case, and positive stain was defined as focal or greater. Statistical differences in paired distributions of UP2 and UP 3 staining intensity and proportion were tested by the Wilcoxon matched-pairs test using PRISM (GraphPad Software, La Jolla, CA, USA), and differences in paired binary distributions of positive and negative cases were tested by McNemar's test using SPSS version 22 (IBM, Redmond, WA, USA).

\section{Results}

ST AIN PATTERN

Consistent with previous reports, immunostaining for UP3 showed membranous, frequently 'plaque-like' positivity or pericellular membranous positivity, while immunostaining for UP2 showed diffuse membranous and cytoplasmic positivity (see Figure 1A-C). Consistent with previous studies of UP2 in various primary carcinomas, ${ }^{10}$ we found no staining for UP2 or UP3 in non-urothelial lesions in the TMA of 88 diverse neoplasms.

HIGH-GRADE CARCINOMAS INVOLVING THE B L A D DER NECK

We stained whole sections of UC $(n=16)$ and PCa $(n=19)$ involving the bladder neck. UP2 staining showed significantly greater intensity $(P=0.003)$ and proportion $(P=0.03)$ than UP3 (Table 1$)$. UP2 outperformed UP3, with a sensitivity of $63 \%$ versus $19 \%$ and specificity of $95 \%$ versus $100 \%(P=0.02)$. Figure 1D-F shows the single focus of UP2 positivity in PCa of the bladder neck.

HIGH-GRADE CARCINOMAS OF THE UPPER TRACT

We stained a previously reported TMA $^{11}$ of highgrade upper tract UCs $(n=19)$ and renal cell carcinomas (clear cell, $n=21$ and papillary, $n=21$ ), CDCs $(n=7)$, and renal medullary carcinomas $(n=2)$, as well as additional whole sections of high-grade upper tract UCs $(n=10)$ and CDCs $(n=5)$. In UC cases, UP2 showed significantly greater intensity and proportion positive (both $P<0.001$ ), consistent with increased sensitivity of $68 \%$ versus $23 \%$ and equal specificity of $100 \%(P=0.006)$ (Table 1$)$.

\section{MET AST A TIC UROTHELIAL CAR CIN OMA}

We stained 30 whole sections of metastatic urothelial carcinomas, including metastases to lymph nodes $(n=11)$, lung $(n=8)$, liver $(n=2)$, stomach/bowel $(n=4)$, bone/soft tissue $(n=4)$ and brain $(n=1)$. As before, UP2 staining showed greater intensity and proportion than UP3 (both $P<0.001$ ), consistent with higher sensitivity $(73 \%$ versus $37 \%$, respectively, $P=0.001$ ); see Table 1 . Neither UP2 nor UP3 showed a significant difference in staining between systemic and nodal metastases $(P=1.0$ and $P=0.43$, respectively). Figure $1 \mathrm{G}-\mathrm{I}$ demonstrates representative staining in a metastatic UC.

\section{VARIANTS OF UROTHELIAL CARCINOMA}

As a descriptive study, we also stained a total of 27 rare variants of UC from the lower tract, finding a significantly greater intensity and proportion positive (both $P<0.001$ ) across cases for UP2 versus UP3, with significantly greater sensitivity $(70 \%$ versus $19 \%$, respectively, $P=0.001$ ). UP2 positivity was predominant in plasmacytoid UC (seven of seven), as well as nested variant (five of five), with one of two cases of each of the clear cell and small cell variants. Single cases of micropapillary, villoglandular and lymphoepithelioma-like UC were each positive, as was UC with syncytiotrophoblastic giant cells. Sarcomatoid (none of three) and 'chordoid' UC (none of two) were uniformly negative, as was a case of UC with giant-cell rich stromal reaction. Figure 1J-L shows representative staining of UC variants.

\section{Discussion}

Proving urothelial origin for a newly diagnosed carcinoma has remained a challenge in surgical pathology, despite improved markers for demonstrating this lineage. ${ }^{8,9}$ We conjecture that this may reflect the uniquely 'plastic' differentiation of the urothelium, as a histological intermediate between squamous and columnar epithelia, capable of a remarkable degree of metaplastic change in physiological, reactive and 
Table 1. Comparison of uroplakin II (UP2) and uroplakin III (UP3) in diagnostically challenging settings

\begin{tabular}{|c|c|c|c|c|c|c|c|c|c|c|c|c|c|}
\hline \multirow[b]{2}{*}{ Diagnostic scenario } & \multirow[b]{2}{*}{ Epitope } & \multirow[b]{2}{*}{ Positivity (\%) } & \multirow[b]{2}{*}{$P$-value* } & \multicolumn{4}{|c|}{ Intensity } & \multirow[b]{2}{*}{$P$-valuet } & \multicolumn{4}{|c|}{ Proportion } & \multirow[b]{2}{*}{$P$-valuet } \\
\hline & & & & 0 & $1+$ & $2+$ & $3+$ & & 0 & $1+$ & $2+$ & $3+$ & \\
\hline \multicolumn{14}{|l|}{ Bladder neck } \\
\hline \multirow[t]{2}{*}{ UC } & UP2 & $10 / 16(62.5)$ & 0.02 & 6 & - & 3 & 7 & 0.003 & 6 & 1 & 3 & 6 & 0.03 \\
\hline & UP3 & $3 / 16(18.8)$ & & 13 & - & 2 & 1 & & 13 & 1 & - & 2 & \\
\hline \multirow[t]{2}{*}{$\mathrm{PCa}$} & UP2 & $1 / 19(5.2)$ & & 18 & - & - & 1 & & 18 & 1 & - & - & \\
\hline & UP3 & 0/19 (0) & & 19 & - & - & - & & 19 & - & - & - & \\
\hline \multicolumn{14}{|l|}{ Upper tract } \\
\hline \multirow[t]{2}{*}{ UC } & UP2 & $17 / 25(68)$ & 0.006 & 8 & 7 & 4 & 6 & $<0.001$ & 8 & 6 & 9 & 2 & $<0.001$ \\
\hline & UP3 & $6 / 26(23.1)$ & & 20 & 5 & 1 & - & & 20 & 4 & 2 & - & \\
\hline \multirow[t]{2}{*}{ Non-UC } & UP2 & $0 / 50(0)$ & & 50 & - & - & - & & 50 & - & - & - & \\
\hline & UP3 & $0 / 49(0)$ & & 49 & - & - & - & & 49 & - & - & - & \\
\hline \multicolumn{14}{|l|}{ Metastatic UC } \\
\hline UP2 & & $22 / 30(73.3)$ & 0.001 & 8 & 3 & 11 & 8 & $<0.001$ & 8 & 4 & 8 & 10 & $<0.001$ \\
\hline UP3 & & $11 / 30(36.7)$ & & 19 & 8 & 2 & 1 & & 19 & 8 & 3 & - & \\
\hline
\end{tabular}

Pattern of variant differentiation in urothelial carcinoma

\begin{tabular}{|c|c|c|c|c|c|c|c|c|c|c|c|c|c|}
\hline \multirow[t]{2}{*}{ Plasmacytoid } & UP2 & $7 / 7$ (100) & 0.001 & - & 1 & 1 & 5 & $<0.001$ & - & 1 & 1 & 5 & $<0.001$ \\
\hline & UP3 & $1 / 7(14.2)$ & & 6 & 1 & - & - & & 6 & - & - & 1 & \\
\hline \multirow[t]{2}{*}{ Nested } & UP2 & $5 / 5(100)$ & & - & 1 & - & 4 & & - & 1 & 2 & 2 & \\
\hline & UP3 & $2 / 5(40)$ & & 3 & 2 & - & - & & 3 & - & 1 & 1 & \\
\hline \multirow[t]{2}{*}{ Sarcomatoid } & UP2 & $0 / 3(0)$ & & 3 & - & - & - & & 3 & - & - & - & \\
\hline & UP3 & $0 / 3(0)$ & & 3 & - & - & - & & 3 & - & - & - & \\
\hline \multirow[t]{2}{*}{ Chordoid } & UP2 & $0 / 2(0)$ & & 2 & - & - & - & & 2 & - & - & - & \\
\hline & UP3 & $0 / 2(0)$ & & 2 & - & - & - & & 2 & - & - & - & \\
\hline \multirow[t]{2}{*}{ Clear cells } & UP2 & $1 / 2(50)$ & & 1 & - & - & 1 & & 1 & - & - & 1 & \\
\hline & UP3 & $0 / 2(0)$ & & - & - & - & - & & - & - & - & - & \\
\hline \multirow[t]{2}{*}{ Small cell } & UP2 & $1 / 2(50)$ & & 1 & 1 & - & - & & 1 & 1 & - & - & \\
\hline & UP3 & $0 / 2(0)$ & & - & - & - & - & & - & - & - & - & \\
\hline
\end{tabular}

UC, Urothelial carcinoma; PCa, prostatic carcinoma.

${ }^{*} P$-value for test for difference in distributions of binary (positive/negative) UP2 or UP3 status in indicated cases, McNemar's test.

† $P$-value for difference in paired distributions in ordinal intensity and proportion scores of UP2 and UP3 stains, Wilcoxon's matched-pairs test.

neoplastic conditions. Until the recent introduction of antibodies against UP3 with properties serviceable for routine diagnostic use, this specific property of urothelial differentiation (urothelial plaques and uroplakin expression) could not be harnessed, and even so has suffered from lack of sensitivity. 
Our findings suggest that detection of the related uroplakin, UP2, provides greater sensitivity with preserved specificity relative to UP3; indeed, of $>160$ non-UC tissues studied, only a single case (a poorly differentiated PCa of the bladder neck) with focal UP2 expression was noted. We note that the superior performance of UP2 is related, to some degree, to the pattern of stain considered positive. The cytoplasmic and membranous stain of UP2 is more diffuse than the "plaque-like' and pericellular membranous stain of UP3; this results in the increased intensity and proportion of staining observed. However, pattern aside, the absolute sensitivity of UP2 was significantly greater than UP3 in each diagnostically challenging setting tested.

Although we would suggest that the primary niche of uroplakins as markers of urothelial histogenesis relates to their high specificity, their sensitivity, particularly that of UP2, deserves consideration in context of the more established urothelial markers. For instance, among upper tract UCs, where we saw a sensitivity of $68 \%$ for UP2 (23\% UP3), both are substantially less than the $100 \%$ sensitivity for p63 and $94 \%$ sensitivity of high molecular weight cytokeratin (HMWCK) seen in the same cohort and reported previously ${ }^{11}$ (with the caveat that HMWCK staining was also positive in $33 \%$ of CDCs and $50 \%$ of medullary carcinomas in the cohort). Although p63 was also $100 \%$ specific in this same TMA cohort, p63 positivity has been seen infrequently in $\mathrm{CDCs},{ }^{12}$ and would be expected (as would HMWCK expression) in primary and metastatic squamous lesions. Similarly, GATA3 expression, although reported to be more sensitive $(88 \%)$ for upper tract UCs than the $68 \%$ we saw for UP2, has been seen in $6 \%$ of $\mathrm{CDCs}^{13}$ and more than half of chromophobe renal cell carcinomas, ${ }^{3}$ paragangliomas ${ }^{14}$ and metastatic breast cancers and other lesions ${ }^{3}$ that may be sampled in the upper tract. In the case of metastases, recent data support the sensitivity of GATA3 $\left(93 \%{ }^{15}\right.$ compared to our $73 \%$ for UP2), with the same caveats regarding its expression by squamous and other lesions. ${ }^{3,16,17}$ Similarly, in the bladder neck, our observation of a sensitivity of $63 \%$ for UP2 staining is inferior to that reported for HMWCK $(97 \%)$ and p63 $(92 \%),{ }^{18}$ or even GATA3 $3{ }^{16}$ with the caveat that these markers are only specific versus PCa but not versus squamous or other lesions in the bladder neck differential. ${ }^{14,16}$ In fact, given the advantageously different subcellular staining patterns for GATA3 and UP2, one might propose that these two markers be used as a "urothelial cocktail' for biopsies, cytology blocks or other settings with limiting diagnostic material. Our group are undertaking preliminary efforts in this regard.
Thus, in each of the diagnostic settings we tested, the result is the same: UP2 outperforms UP3 in terms of sensitivity, while both are highly specific. While it bears consideration that neither are as sensitive as more established markers, our experience is that, other than uroplakins, no markers have a degree of specificity sufficient to confirm the diagnosis. The increased diagnostic performance of UP2 over UP3 may prove to be of great help to surgical pathologists in these diagnostically challenging settings and in the setting of limited tissue samples, where performance of extended immunohistochemical panels is not feasible.

\section{References}

1. Wu XR, Kong XP, Pellicer A, Kreibich G, Sun TT. Uroplakins in urothelial biology, function, and disease. Kidney Int. 2009; 75; 1153-1165.

2. Higgins JP, Kaygusuz G, Wang L et al. Placental s100 (s100p) and GATA3: markers for transitional epithelium and urothelial carcinoma discovered by complementary DNA microarray. Am. J. Surg. Pathol. 2007; 31; 673-680.

3. Miettinen M, McCue PA, Sarlomo-Rikala M et al. GATA3: a multispecific but potentially useful marker in surgical pathology: a systematic analysis of 2500 epithelial and nonepithelial tumors. Am. J. Surg. Pathol. 2013; 38; 13-22.

4. Gandou C, Harada K, Sato Y et al. Hilar cholangiocarcinoma and pancreatic ductal adenocarcinoma share similar histopathologies, immunophenotypes, and development-related molecules. Hum. Pathol. 2013; 44; 811-821.

5. Kaufmann O, Volmerig J, Dietel M. Uroplakin III is a highly specific and moderately sensitive immunohistochemical marker for primary and metastatic urothelial carcinomas. Am. J. Clin. Pathol. 2000; 113; 683-687.

6. Brown HM, Wilkinson EJ. Uroplakin-III to distinguish primary vulvar Paget disease from Paget disease secondary to urothelial carcinoma. Hum. Pathol. 2002; 33; 545-548.

7. Mhawech P, Uchida T, Pelte MF. Immunohistochemical profile of high-grade urothelial bladder carcinoma and prostate adenocarcinoma. Hum. Pathol. 2002; 33; 1136-1140.

8. Parker DC, Folpe AL, Bell J et al. Potential utility of uroplakin III, thrombomodulin, high molecular weight cytokeratin, and cytokeratin 20 in noninvasive, invasive, and metastatic urothelial (transitional cell) carcinomas. Am. J. Surg. Pathol. 2003; 27; 1-10.

9. Gruver AM, Amin MB, Luthringer DJ et al. Selective immunohistochemical markers to distinguish between metastatic highgrade urothelial carcinoma and primary poorly differentiated invasive squamous cell carcinoma of the lung. Arch. Pathol. Lab. Med. 2012; 136; 1339-1346.

10. Hoang L, Qi W, Bremer R et al. A newly developed anti-uroplakin II monoclonal antibody with increased sensitivity in urothelial carcinoma of the bladder. Mod. Pathol. 2013; 26(Suppl 2); $1 \mathrm{~A}-534 \mathrm{~A}$.

11. Carvalho JC, Thomas DG, McHugh JB, Shah RB, Kunju LP. P63, CK7, PAX8 and INI-1: an optimal immunohistochemical panel to distinguish poorly differentiated urothelial cell carcinoma from high-grade tumours of the renal collecting system. Histopathology 2012; 60; 597-608. 
12. Gupta R, Billis A, Shah RB et al. Carcinoma of the collecting ducts of Bellini and renal medullary carcinoma: clinicopathologic analysis of 52 cases of rare aggressive subtypes of renal cell carcinoma with a focus on their interrelationship. Am. J. Surg. Pathol. 2012; 36; 1265-1278.

13. Gonzalez-Roibon N, Albadine R, Sharma R et al. The role of GATA binding protein 3 in the differential diagnosis of collecting duct and upper tract urothelial carcinomas. Hum. Pathol. 2013; 44; 2651-2657.

14. So JS, Epstein JI. GATA3 expression in paragangliomas: a pitfall potentially leading to misdiagnosis of urothelial carcinoma. Mod. Pathol. 2013; 26; 1365-1370.

15. Zhao L, Antic T, Witten D et al. Is GATA3 expression maintained in regional metastases? A study of paired primary and metastatic urothelial carcinomas. Am. J. Surg. Pathol. 2013; 37; 1876-1881.
16. Chang A, Amin A, Gabrielson E et al. Utility of GATA3 immunohistochemistry in differentiating urothelial carcinoma from prostate adenocarcinoma and squamous cell carcinomas of the uterine cervix, anus, and lung. Am. J. Surg. Pathol. 2012; 36; $1472-1476$.

17. Gulmann C, Paner GP, Parakh RS et al. Immunohistochemical profile to distinguish urothelial from squamous differentiation in carcinomas of urothelial tract. Hum. Pathol. 2013; 44; 164 172.

18. Kunju LP, Mehra R, Snyder M, Shah RB. Prostate-specific antigen, high-molecular-weight cytokeratin (clone 34betae12), and/or p63: an optimal immunohistochemical panel to distinguish poorly differentiated prostate adenocarcinoma from urothelial carcinoma. Am. J. Clin. Pathol. 2006; 125; 675681. 